\section{Brain biopsy and a case of dementia that wasn't}

Dear Editor - This is a case report of a patient incorrectly diagnosed with dementia with lewy bodies (DLB) principally on the basis of a brain biopsy. Review of the case revealed that he had none of the core clinical features of DLB and that the history of cognitive decline was less than six months. This case highlights a number of issues, most notably that dementia is a clinical diagnosis; a good history is most important in making the diagnosis of dementia and investigations are not an adequate substitute. This case also demonstrates similarities between DLB and delirium.

\section{Case history}

$\mathrm{JF}$, a 56 year old man presented to A\&E in a dishevelled, agitated state claiming he was 'going to die'. He appeared tense and anxious. His speech was low in volume with evidence of perseveration. There was paucity of thought and his thought content was pessimistic. His only physical complaint was constipation. He was disorientated in time. Other than mild pyrexia his physical examination was unremarkable. In view of his confusional state he was admitted for medical assessment. Laboratory tests including a full blood count, urea and electrolytes, liver function, autoantibody screen, vitamin B12, folate, thyroid function tests, syphilis serology were normal. An ultrasound scan of abdomen was normal. Psychiatric consultation while a medical inpatient revealed a six week history of low mood and social withdrawal. A decline in his daily functioning was evidenced by working less and interacting less. He was described as having been inactive and avoidant of eye contact in recent weeks. Poor sleep, poor appetite and some weight loss were also noted. JF described feeling 'stressed' and expressed concern about his physical health. He felt unable to think clearly. He expressed a passive death wish. A number of somatic preoccupations such as believing he was 'brain damaged', 'kidneys are failing', 'heart has stopped' and that he 'is dying' were noted. He also expressed some vague paranoid ideas.

JF's background history revealed that he was a poor scholar, who worked as a farm labourer. He was unmarried and had never had an intimate relationship. He had no past psychiatric history nor was there any family history of mental illness. On mental state examination he was agitated. There was evidence of psychomotor retardation. He displayed poverty of speech. His thoughts revealed nihilistic and paranoid ideation. He had poor concentration. It was noted that he was of 'possible borderline IQ' though this was not formally assessed. The initial psychiatric consultation opinion was of a severe depressive episode. JF was transferred to the psychiatric admission ward.

Following little response to the antidepressant mirtazapine, his treatment was changed to lofepramine. He showed little improvement over the next month. Due to the resistant nature of his depression, lithium augmentation was introduced with marginal benefit. At this point ECT was considered in view of lack of response to antidepressant medication.

However, over the next couple of weeks a significant decline in JF's cognitive function was noted. He was observed to be withdrawn with little awareness of his surroundings. He scored 11/30 on Mini Mental State Examination. He developed urinary incontinence. In view of his rapid decline a medical consultation was requested. The medical impression at this time was of 'a rapid dementing illness with delirium'. The major concerning features were 'global intellectual decline with basal ganglia signs'. JF was transferred back to the medical ward. Prophylactic treatment with acyclovir was given to cover herpes simplex encephalitis. His condition continued to deteriorate. He was observed to be toe walking, doubly incontinent and exhibiting aggressive behaviour. At times he was found to be mute, vague and disorientated.

Investigations at this time showed a mildly elevated white cell count and slightly abnormal liver function which settled quickly. Brucella, leptospirosis titres, ANA, rheumatoid factor and ANCA were all negative. Viral hepatitis screen was negative. Ceruloplasmin was normal. Mantoux test was negative. A chest X-ray was normal. MRI and CT brain did not reveal any abnormality. Functional scanning was not carried out. Lumbar puncture revealed mildly elevated protein in the CSF. EEG showed non-specific bilateral temporal lobe disturbance.

Due to the diagnostic dilemma and the rapid deterioration in his condition, JF was transferred to a tertiary centre for further investigation including a brain biopsy. The brain biopsy showed frontal lobe lewy body accumulation in the cortex. An exaggerated response to antipsychotic medication was noted during his admission. Neurological opinion was that this finding in addition to his fluctuating mood and behaviour and the brain biopsy report supported a diagnosis of DLB.

After the diagnosis of DLB was made, JF was admitted to a long-term care unit and discharged from psychiatric care. He had a psychiatric review following a re-referral six years later. It was found there had been no decline in his cognitive function. There was no evidence of Parkinsonism. He was continent of both urine and faeces. There was no history of falls, fluctuating consciousness or visual hallucinations. Neuropsychological assessment following re-referral concluded that JF "had a long-standing history of moderate learning disability" and that "current deficits in functioning are reflective of cognitive impairment due to his learning disability".

The brain biopsy was reviewed by the neuropathologist with alpha syneuclein antibody which was not available at the time of the original biopsy. This review concluded "I cannot confirm the presence of lewy bodies by immunochemistry and as such the original diagnosis cannot be substantiated." It was concluded by psychiatry that the initial diagnosis was not DLB or a dementia of any type. In retrospect, the probable diagnosis was a delirium in a man who had underlying learning disability. The cause of the delirium is unknown.

\section{Discussion}

This case highlights a number of issues about dementia in general and DLB in particular. Firstly dementia is a clinical diagnosis; history, including collateral history, is the most important factor in making the diagnosis. Secondly, DLB has many features similar to or which overlap with delirium, making it difficult to distinguish between these, particularly on initial assessment. ${ }^{1}$ Thirdly, brain biopsy or other highly technical or invasive procedures are no substitute for the appropriate history in making a diagnosis of dementia. 
Dementia is a syndrome due to disease of the brain, usually of a chronic or progressive nature, in which there is a disturbance of multiple higher cortical functions including memory, thinking, orientation, comprehension, calculation, learning capacity, language and judgement. This must have been present clearly for at least six months. Consciousness is not clouded. The impairments of cognitive function are commonly accompanied, and occasionally preceded, by deterioration in emotional control, social behaviour or emotion. Diagnosis of dementia should be deferred in the presence of an acute confusional state. ${ }^{2}$ In our case the history was only six weeks and without a longer history the diagnosis of dementia was less likely.

DLB (dementia with lewy bodies) is a major cause of dementia in the elderly. It is considered the second commonest cause of degenerative dementia in old age after Alzheimer's disease. Consensus criteria for the clinical diagnosis of probable and possible DLB are as follows: The central feature required for a diagnosis of DLB is progressive cognitive decline of sufficient magnitude to interfere with normal social or occupational function. Two of the following core features are essential for a diagnosis of probable DLB, one is essential for possible DLB:

- Fluctuating cognition with pronounced variations in attention and alertness

- Recurrent visual hallucinations which are typically well formed and detailed

- Spontaneous motor features of Parkinsonism.

Features supportive of the diagnosis include repeated falls, syncope, transient loss of consciousness, neuroleptic sensitivity, systematised delusions and hallucinations in other modalities. ${ }^{3}$ The main differential diagnoses to be considered are delirium secondary to an underlying physical illness or multi-infarct dementia. Our case did not have the clinical features to fit the consensus criteria for DLB and the differential diagnosis of delirium seems more likely.

The role of brain biopsy in dementia is uncertain. In general, it is regarded as a procedure of last resort, undertaken on the basis of a clinical risk:benefit analysis where the probability of making a diagnosis and altering management is seen to outweigh the risks of possible serious complications of this invasive procedure. It is generally considered in younger patients where clinical presentation or investigations suggest a reversible cause such as an inflammatory process. In a number of studies, specific diagnosis was achieved in $22-84 \%$ of cases, most commonly Alzheimer's disease and CJD. ${ }^{4}$ The value of brain biopsy for accurate diagnosis in dementia is in distinguishing reversible from irreversible causes which can be difficult on clinical grounds alone. A specific diagnosis can be established in over $50 \%$ of cases of dementia coming to brain biopsy and a treatable cause identified in about $10 \% .^{4}$

Complicating the diagnosis of DLB is the non specificity of lewy bodies for DLB. ${ }^{5}$ One histopathological review of 100 clinically diagnosed Parkinson's disease patients revealed cortical lewy bodies in all cases. In another study, lewy bodies were found in $12 \%$ of autopsy cases carrying a clinical diagnosis of Alzheimer's disease. ${ }^{5}$ Cortical lewy bodies are typically found in DLB and Parkinson's disease, they are also found in other neurological disorders. ${ }^{6}$ Lewy bodies have been found in only $2.3 \%$ of non demented elderly. ${ }^{7}$
Consensus clinical diagnostic criteria for DLB have been validated against neuropathologic autopsy findings. Mega and colleagues ${ }^{7}$ and McKeith et al $^{10}$ found sensitivity for a clinical diagnosis of DLB at $75 \%$ and $79 \%$ versus specificity at $83 \%$ and $95 \%$ respectively - levels of diagnostic accuracy that are similar to those for Alzheimer's disease and vascular dementia. False positive diagnoses of DLB do not appear to be as frequent as false negatives. ${ }^{8}$ This is exemplified in the study by Lopez et $\mathrm{al}^{9}$ where the clinical diagnosis was compared with the neuropathological diagnosis; the sensitivity for the clinical diagnosis for DLB was $30.7 \%$ and the specificity was $100 \%$. This prospective analysis of the clinical criteria for DLB reveals low sensitivity and high specificity, consistent with several retrospective clinicopathological studies. ${ }^{9}$

Current clinically based diagnostic criteria for DLB have limited accuracy. A revision of the International Consensus Criteria for DLB has recommended that low dopamine transporter uptake in the basal ganglia, as shown by SPECT or PET imaging, be a suggestive feature for diagnosis. This was confirmed in a recent study by the DLB Study Group which confirmed the high correlation between abnormal DAT activity measured with (123)I-FP-CIT SPECT and a clinical diagnosis of probable DLB. The diagnostic accuracy is sufficiently high for this technique to be clinically useful in distinguishing DLB from Alzheimer's disease. ${ }^{10}$

These advances highlight the ongoing efforts to improve the accuracy of diagnosis in DLB. This case reminds us that despite such developments, a convincing history is vital in making the diagnosis of dementia, more important than a highly invasive procedure such as a brain biopsy.

\section{Conclusion}

This case demonstrates the enduring importance of a careful history in modern medical practice especially in the diagnosis of a chronic illness such as dementia. Also it reminds us how delirium can present with features of DLB.

*Clare O'Toole, Senior Registrar in Psychiatry, Clondalkin Mental Health Service, Dublin 22

Brenda Wright, Consultant Forensic Psychiatrist, National Forensic Mental Health Service, Central Mental Hospital, Dundrum, Dublin 14

Feargal Leonard, Consultant Psychiatrist, Dept of Old Age Psychiatry, Cavan General Hospital, Cavan Vincent Russell, Consultant Pstchiatrist, Dept of Psychiatry, Cavan General Hospital, Cavan, Ireland.

References

1. Robinson MJ. Probable Lewy Body Dementia Presenting as Delirium. Psychosomatics 2002; 43, 84-86.

2. The ICD-10 Classification of Mental and Behavioural Disorders 1992. World Health Organisation. Geneva. p45-46.

3. McKeith IJ. Dementia with Lewy bodies. Br J Psychiatry 2002; 180, 144-147

4. Warren JD, Schott JM, Fox NC, Thom M, Revesz T, Holton JL, Scaravilli F, Thomas DGT, Plant GT, Rudge P, Rossor MN. Brain biopsy in dementia. Brain 2005; 128, 2016 2025.

5. Papka M, Rubio A, Schiffer RB. A Review of Lewy Body Disease, an Emerging Concept of Cortical Dementia. Journal of Neuropsychiatry 1998; 10 (3), 267-279. 6 . Burn DJ. Cortical Lewy body disease. Journal of Neurology, Neurosurgery, and Psychiatry 2004;75:175-178

7. Mega MS, Masterman DL, Benson DF et al. Dementia with Lewy Bodies: Reliability and validity of clinical and pathologic criteria. Neurology 1996; 47, 1403-1409.

8. McKeith IG. Dement Geriatr Cogn Disord 2004; 17 (suppl 1): 1-2

9. Lopez OL, Becker JT, Kaufer DI et al. Research Evaluation and Prospective Diagnosis of Dementia with Lewy Bodies. Arch Neurol 2002; 59:43-46.

10. McKeith I, O'Brien J, Walker Z, Tatsch K, Booij J, Darcourt J, Padovani A, Giubbini R, Bonuccelli U, Volterrani D, Holmes C, Kemp P, Tabet N, Meyer I, Reininger C; DLB Study Group. Sensitivity and specificity of dopamine transporter imaging with 123l-FP. CIT SPECT in dementia with Lewy bodies: a phase III, multicentre study. Lancet Neurol. 2007; 6(4): 305-13. 